\title{
Making DAB Work: New Opportunities for Digital Radio in Europe
}

Lawrie Hallett

\section{INTRODUCTION}

Digital Audio Broadcasting (DAB) is now commonplace in a number of European jurisdictions. However, in the greater scheme of things, its success, when compared to early predictions, has been somewhat limited. Technologies it was intended to supplant (most notably analogue FM broadcasting) continue to thrive, whilst alternative platforms, such as the smartphone and connected speakers (e.g. Amazon's Alexa and Google Home) have recently gained rapid mass acceptance. In addition, new programme delivery methods (podcasting, and "listen again" services etc.) within the online environment, have emerged to further diversify the reception options available.

Although such developments are, doubtless, complicating factors, this chapter suggests that it is not these that are primarily responsible for limiting DAB's wider international success to date. Rather, it is argued, the key issue is DAB's historic inability to provide an all-encompassing transmission platform, suitable for use across the entire broadcast radio sector. Bluntly put, DAB has not been seen as a comprehensive replacement technology. Unlike FM broadcasting before it, DAB has yet to prove itself capable of meeting the needs of all types of radio broadcaster, that is to say from the largest public service operators and commercial concerns, right through to the various smaller commercial and not-for-profit community radio service providers.

A key difficulty with the technology has been its historical inability to deliver small-scale broadcast coverage on a cost-effective basis. In addition, such a lack of flexible scalability in terms of coverage has tended to be reinforced by DAB coverage planning approaches and spectrum occupancy plans that have, perhaps understandably, or even conveniently, assumed minimal use of the platform by such smaller operators. 
Beginning with an historical examination of the development of DAB, this chapter both explores such difficulties and focuses on current approaches intended to improve upon the technology's suitability for smaller scale broadcasting operations. In particular, consideration is given to the recent emergence of alternative technological and regulatory approaches that may yet see DAB delivered in ways that both expand and enhance opportunities right across the broadcast radio sector in all its increasing diversity.

Although regulatory frameworks vary from jurisdiction to jurisdiction, an almost universal truth is that demand for broadcast licences outstrips their supply. In many countries, this is particularly acute at the small scale, for community based services and smaller commercial stations. Availability of DAB for such operators is one issue for them, historically, the other has been cost.

In terms of technology, the past decade has seen a confluence of conditions that have made lower-cost approaches to DAB delivery more practical. Open source DAB software emerged to take advantage of the falling cost and rising capabilities of digital hardware. Rather than use expensive bespoke hardware solutions, it is now possible to use generic computer hardware in conjunction with somewhat more specialist (but nevertheless readily available and relatively inexpensive) programmable hardware, in the form of a, so-called, software defined radio (SDR) to generate and transmit DAB signals. Initially, however, such nascent technological advantages had a minimal impact. It took time for the radio industry to realise that they allowed for a use of DAB that had not previously been assumed possible. Only when individual national regulators, such as Ofcom in the United Kingdom, began to allow field trials did the potential material impacts of such new approaches begin to become clearer.

For smaller scale community radio services and commercial radio stations, it is now apparent that the potential of these new approaches to DAB could be profound. Suddenly, cost effective options for digital broadcasting alongside larger stations may be possible. The alternative, that smaller operators could instead be left in a so-called "analogue backwater" on FM or AM (when all major broadcasters may have abandoned such platforms in favour of a digital-only future), now seems, potentially at least, a little less likely. The resultant challenge, for governments and regulators alike, is how to develop legal and regulatory environments that can best facilitate a broader digital uptake.

\section{DAB AS TECHNOLOGY}

Historically, DAB has always been a transnational project. The underlying objectives that drove the development of DAB at the outset were focused on the technical modernisation and improvement of broadcast radio delivery. The European Union-backed research project behind DAB, known as Eureka 147 
(Bower 1998), was formed in order to develop a programme platform technology capable of delivering such changes. The project drew in both broadcasters and technology companies from a variety of European countries and was quickly embraced by others outside the European Union, including, for example, the Canadian Research Council (more of which later).

Specifically, back in the 1980 s, the fundamental issue was capacity; demand for additional radio broadcasting licences could not be met, simply because, using the existing technologies of the day, there was insufficient radio frequency capacity within which to accommodate new services. The goal, therefore, was to develop a broadcasting platform that would deliver greater spectral efficiency and, additionally, improve audio quality and resistance to interference, as well as enhance operational flexibility. Such improvements over traditional analogue broadcasting platforms (AM and FM) would provide for the expansion of the medium, directly benefiting both broadcasters and listeners alike.

\section{The "Hype" of New Technologies}

Since its introduction the view of DAB and the degree to which this technology has delivered against its early objectives have largely followed a recognisable trajectory, what has become known as the "hype cycle" (see, for example, Fenn/ Raskino 2008; Gartner 2017). Originally developed by the business consultancy firm Gartner, the hype cycle hypothesises that any new technology results from a "technological trigger" (in this case the need to improve the carrying capacity of broadcast radio transmission systems). Thereafter, the theory suggests that a new technology will go through distinct phases: a "peak of inflated expectations" and "a trough of disillusionment" before reaching a "slope of enlightenment" and then, hopefully, "a plateau of productivity" (ibid.).

This conceptual cycle can be criticised, not least because of its use of subjective terminology. However, for DAB at least, it does appear possible to map the emergence of the technology to the various phases of the cycle. Indeed, a more detailed breakdown of the hype cycle (Crawford 2013) identifies further traits, such as the role of early adopters, mass media promotion and negative media coverage, each of which can be similarly mapped against the historical development of DAB. It is, therefore, arguable that the underlying proposition of the hype cycle may have some validity (at least in this instance). Thus, it may also be useful in providing some contextual understanding as to how DAB came to be positioned as it is today. Smaller scale broadcasters (both commercial and community based) have historically tended towards a negative view of DAB, both on ground of cost and, more fundamentally, in relation to the standard's seeming inability to deliver more tightly focused geographical coverage. Without access to DAB coverage of relevant scale at manageable cost, smaller opera- 
tors understandably fear the emergence of a potentially dominant broadcasting platform, from which they would effectively be excluded. Their views of DAB's hype cycle and indeed their impact on its development are therefore both relevant and particular.

Having been in existence for some three decades, DAB might by now be expected to be well into its productivity phase; however, in some ways at least, it still appears to be climbing the slope of enlightenment towards this goal. There are various reasons for this lack of clarity, not least the fact that different jurisdictions have tackled the introduction of DAB in different ways and with differing degrees of enthusiasm. Successes in some countries have often been tempered by failures in others.

As DAB technology has gradually matured, both its benefits and its weaknesses have become increasingly apparent. Moreover, analysing the impacts of DAB roll-out quickly suggests that the distribution of its benefits and the impact of its weaknesses has not been spread equally across all sectors of the broadcast radio industry. A brief examination of DAB's origins soon suggests why this might be the case.

\section{The Early History of DAB - Inflated Expectations?}

Back in the late 1980 s when the original Eureka-147 Digital Audio Broadcasting standard was first conceived, the world of radio broadcasting was a very different place (Kozamernik 1995; O’Neill 2009). Key differences were not just technological; they were fundamentally structural, concerning both economics and regulation, reflecting the degree to which the zeitgeist has changed over the intervening years. The number of broadcast radio stations operating was considerably smaller, with commercial radio still expanding in many countries and with community radio services much less pervasive than they are today. Moreover, the economics of the commercial element of the industry in particular tended towards being both more heavily regulated and less conglomerated. Despite subsequent commercial conglomeration, over the intervening years the industry as a whole has become increasingly diverse, particularly where a rapid expansion of smaller scale community radio services has since taken place.

When the European Union agreed to fund the original Eureka-147 research project, such small-scale operations were far from a priority. Proponents of the new digital radio broadcasting standard were composed primarily of larger European public service broadcasting (PSB) organisations, along with research institutions with a particular interest and expertise in digital audio and radio frequency transmissions. Meeting the demands of large-scale radio broadcasters, both public service and commercial, (making room for additional services and improving coverage etc.), was the priority. Thus, the emergent technical 
standard came to reflect this reality and subsequent frequency planning and regulatory approaches in support of the standard did the same.

In order to achieve its objectives, the Eureka 147 project team designed a system that had some characteristics that were very different from those of traditional analogue radio transmissions. The primary difference was the use of a multiplex, combining multiple stations into a single transmitted signal, for separation and selection by the receiver. A larger block of radio frequency spectrum is required for such an approach, however not only does it allow for a greater number of services to be broadcast within a defined amount of radio spectrum (when compared to FM transmissions), but such an approach also provides intrinsically enhanced resistance to interference.

For PSB and larger commercial operators, the advantages of digital radio are generally, and quite obviously, realised by the DAB standard. Fundamentally, the benefits centre around improved spectral efficiency, creating the ability to carry a greater number of services within a given amount of radio frequency spectrum (so, for example, the BBC could add Six Music, Radio 1 Extra and Radio 4 Extra, as well as the Asian Network, plus the BBC World Service to its roster of services broadcast across the United Kingdom). For politicians and regulators, the greater spectral efficiency of DAB, realised in particular for networked services that can re-use the same frequency allocation multiple times (in what is known as a Single Frequency Network [SFN]), was also a material prize.

\section{Relevance to Smaller Radio Stations}

The benefits summarised above are, however, of little relevance to the majority of smaller broadcast radio stations, typically employing a single transmitter for their operations. For such broadcasters, there are two key problems with the use of DAB broadcasting. First, there is the issue of cost. Historically, since DAB has been introduced, capital costs of equipment and, in particular, recurrent operational costs, have been higher for DAB than those required for equivalent coverage using the traditional analogue FM platform. Second, there is the matter of access, in particular defined by spectrum planning approaches, which, historically and for the reasons discussed above, has rarely prioritised, or really even seriously considered, the economic and operational requirements of smaller broadcasters.

Such difficulties aside, DAB still offers one overarching potential benefit when compared to analogue broadcasting: its service-carrying capacity. The limited amount of analogue radio frequency spectrum available for radio broadcasting means such resources have not been able to meet the continuing high levels of demand for additional radio broadcasting licenses. By bringing new frequency allocations into broadcasting use, and by using these efficiently, 
digital broadcasting technologies, such as DAB, offer a way to reduce this longstanding imbalance between supply and demand.

\section{Emergent Shortcomings - Disillusionment?}

In 1997, the Eureka-147 Project Office published an overview of the standard in what has now become a useful historical document, 'Eureka-147 - Digital Audio Broadcasting', which is still available online (Eureka 1997). The opening page of the project office publication states:

Digital Audio Broadcasting, DAB, is the most fundamental advance in radio technology since the introduction of FM stereo radio. It gives listeners interference-free reception of highquality sound, easy-to-use radios, and the potential for wider listening choice through many additional stations and services. (ibid.: 2 )

Referring back to the hype-cycle, clearly, such statements can be thought of as being typical of the initial "expectation" phase, which arguably, in the case of $\mathrm{DAB}$, can broadly be thought of as covering the 1990s. The above statement is representative of much of the writing about DAB at that time, highlighting benefits alone without reference to any of the system's operational limitations, in terms of issues such as scale and cost.

Around this period, proponents of the standard not only promoted the technology's functional capabilities, but they also made various wider ambitious claims about its potential to change the broadcast radio ecology. David Withero, the then president of EuroDAB (the then European DAB Forum), interviewed for the US industry journal, Radio World (RW) optimistically suggested that the technology was "on its way to becoming - if it is not already - a world standard for digital radio" (Clark 1996: 1).

Withero's comment came approximately a year after the start of regular networked DAB broadcasting in Norway (NRK 2017), Sweden (Sveriges Radio 2008) and the United Kingdom (Withero/Lavern 1995) during the Summer and Autumn of 1995. Even then, however, the potential for "disillusionment" was already apparent. The USA was fast back-tracking from its earlier commitment to the standard, instead beginning the development of what would eventually become HD Radio, its current domestic digital radio standard (see, for example, Anderson 2013).

By the first decade of this century, DAB had clearly begun to move through the hype cycle, away from the earliest expectation stage towards the disillusionment stage. Along with increased interest from academics in the development and implementation of DAB (see, for example, Lax 2003), it was Community 
Radio campaigners that were some of the first to really identify the limitations of DAB for smaller-scale broadcasters.

Perhaps the earliest comprehensive analysis of the implications of DAB for such operators came from the Netherlands Community Radio support organisation, OLON. "Eureka! Een Oplossing Voor Digitale Kleinschalige Radio" (Eureka! A Solution For Digital Small-Scale Radio), published in 2002 (OLON 2002), identified various issues around the standard and attempted to suggest possible alternative approaches, both within the DAB standard and through the use of alternative digital systems, such as Digital Radio Mondiale (DRM).

In some respects, the OLON publication was ahead of its time, clearly identifying the issues of cost and access, however, the solutions put forward were of somewhat limited relevance. In essence, not only were technological solutions still some years off, but also the operational impacts of DAB were still to be fully understood. Moreover, the driver of so-called "digital switch-over" (a wholesale move away from analogue broadcasting) was, at that time, not given serious credence.

Public awareness of DAB was increasing throughout the first decade of this century, as was the degree of coverage in the press. Critical reporting tended to focus on issues of the availability and reliability of reception (coverage) as well as the audio quality of DAB transmissions (see, for example, Schofield 2006 and Robjohns 2009). It is perhaps not surprising that DAB coverage was perceived as unreliable during its launch phase, not least because network roll-out cannot be completed overnight, as it takes time for any such network to be fully planned and completed. Towards the end of the first decade of this century, the importance of duplicating DAB coverage in such a way as it was perceived to be at least as good as the FM services it was intended to replace had been recognised by both proponents of the standard and by regulators. In the UK, Ofcom (The Office of Communications) made it perfectly clear that British DAB planning would seek to duplicate existing analogue coverage:

As part of the government's Digital Radio Action Plan, Ofcom is currently leading a process to consider the future spectrum planning requirements of digital radio, to prepare for the digital radio upgrade ... This process will establish and agree the current levels of acceptable coverage of $F M$, which will provide the benchmark for all future planning, and determine the most technically efficient way of matching DAB coverage to FM. (Ofcom 2010: 20)

More recently, the European Broadcasting Union, in bringing together recommendations for the successful delivery of digital radio services, noted that: "At the end of the day digital radio coverage must be the same as analogue radio" (EBU 2014: 7). Emphasising the point, the same document added: "Listeners will not accept losing coverage in comparison with analogue services" (ibid.). 
Issues relating to audio quality can perhaps best be understood in the context of early claims that DAB would provide "near-CD quality" (Spikofski and Klar 2003) audio or even “'CD-quality' audio” (O’Neill et. al. 2010: 87). The early days of DAB were:

[A]ccompanied by a tremendous optimism buoyed by its technical achievements concerning the potential for innovative new dimensions to radio as a medium. A key feature ... was its promise of exceptional audio quality. (ibid.)

While it was, and still remains, that case that a DAB multiplex is theoretically capable of delivering a small number audio services of very high audio quality, in practice, such an approach has not been the norm:

More often than not, however, bit-rates are determined by the minimum necessary for acceptable listening, not the maximum or even the recommended levels for effective audio performance. (ibid.)

By sharing out the finite capacity of a given DAB multiplex between a greater number of services, listeners are provided with more choice, albeit at the price of reduced audio fidelity. O'Neill notes the reality that, "broadcasters (and consumers) have tended to prefer quantity over quality” (ibid.).

\section{Finding Solutions - Towards EnLightenment?}

Grant Goddard brings together the various concerns about the DAB standard in his book DAB Digital Radio Licensed to Fail. (Goddard 2010) By the time this was published, regulators had begun to recognise that at least some of the issues identified as problematic with DAB did warrant further examination. In the context of the hype cycle DAB had begun to climb "the slopes of enlightenment". Now, with the advantage of considerable operational experience over some fifteen or so years, the benefits of the standard had become properly understood. However, so too had the material problems that remained to be addressed if DAB was to succeed as a long-term universal digital replacement for analogue broadcasting.

In the UK, after considerable preparation and liaison with Ofcom, the Department for Culture Media and Sport (DCMS) published the first version of its Digital Radio Action Plan (DCMS 2010). This, along with subsequent more detailed work, such as Ofcom's “DAB Coverage Planning Report To Government" (Ofcom 2012) sought to address coverage (and other) concerns, in order to enhance the uptake of DAB. Since 2010, Ofcom's Communications Market 
Digital Radio Report has tracked the uptake of DAB and other forms of digital radio listening (Ofcom 2016a).

From around the same time, the British regulator has also sought to address some of the shortcomings of DAB technology as these relate to the operations of smaller-scale broadcasters. The previously identified issues of cost and relevant coverage were considered key to the regulator's approach. If capital and operational costs could be substantially reduced and if additional localised frequency resources could be identified, might it then be possible to make DAB a viable option for such stations?

\section{SMALL-SCALE DAB TRIALS}

Ofcom began by examining new technical approaches to the delivery of DAB through the use of open-source DAB technology, selecting Central Brighton as the location for initial tests in 2012 (Ray 2013; Mustapha 2013). Operating under a so-called "Test and Development Licence" these transmissions from a single DAB transmitter were not intended for public reception. Rather, they were designed "to inform policy makers of the practicalities of low cost DAB solutions when used to serve small areas" (Mustapha 2013:1), using software based alternatives to traditional transmission infrastructure.

The equipment and systems used to deliver the Brighton transmissions did not simply emerge fully-formed from the UK regulator's radio engineering team. Rather, Ofcom employed open-source technology which had originally been developed by the Communications Research Centre (CRC), a government funded body in Canada, which released its original DAB transmission software under a General Public Licence (GPL) in 2009 (ibid., 6). Although the original CRC project ended some years ago, its work continues to be built upon by Open Digital Radio (ODR), a Geneva-based not-for-profit association, which also has close links to the European Broadcasting Union (EBU), that regularly runs workshops and conferences to promote and develop DAB in all its forms (Coinchon 2010).

It should also be noted that Ofcom was not originally proactive in relation to the initial Brighton test, rather it merely acquiesced to an internal request from an individual employee. That said, once the initial test proved successful, Ofcom did move quickly to further examine possibilities for smaller-scale DAB transmissions with a series of trial broadcasts, this time intended for public reception. The trials, in ten locations across England and Scotland, were supported by the relevant British Government department (DCMS), and each began during 2015. Initially intended to last for only nine months each, in early 2016 Ofcom extended all of the trials for a further two years, such that each would run through into 2018. More recently, with both the UK Government 
and Ofcom struggling to finalise and introduce a new regulatory framework, these trials have been further extended into the Spring of 2020 .

The wider trials have been able to investigate additional elements of the DAB standard. In particular, they have made use of open source approaches to the creation of SFNs (Ofcom 2016b: 4) and have been proactive in the promotion of a newer version of the DAB standard known as DAB+ (see, for example, Herrmann et. al., 2007; and Sabel, 2013). Indeed, the ten localised trial multiplexes have arguably been instrumental in demonstrating the attractiveness of this new version of DAB (Ofcom 2016b: 15). Using DAB+ allows for an increased number of programme services to be carried by a single multiplex at a given perceived audio quality. By extension this means that the operational costs of running a multiplex can be shared between a greater number of stations, thus further tackling the issues around recurrent operational costs for smaller-scale broadcasters.

\section{Consulting on the Future}

The currently extended trials also have wider objectives than those of their predecessor. Beyond further examining the capabilities of software-based DAB transmissions, they are also intended to evaluate possible regulatory approaches to the operation of such services on a permanent basis in future. Ofcom was also keen to gauge the likely demand for such services and to see how such DAB multiplexes might be structured, managed and operated going forward.

In the trial, various operational structures were permitted. Some multiplex operators established themselves as independent commercial, for-profit, companies, others as separate not-for-profit organisations, whilst others still were established within existing community-based or commercial station operating companies. The specific approach to trialling the technology was based on achieving outcomes of relevance to radio broadcasting within the UK. However, the approach to various underlying regulatory issues certainly has wider international relevance. The fact that similar trials have since been adopted in other jurisdictions demonstrates just how similar the underlying issues can be across national borders.

During the experiment each operator was independent from the others, but the question of ownership in the longer term has, for some at least, become a major concern. In British Community Radio, no two stations can be owned by the same company (or other type of body corporate) (HMSO 2004: 4) and individuals may not hold any such licence (ibid.). The objective of this approach is to preserve local ownership and control of such stations. This is something that has almost entirely disappeared from British commercial radio broadcasting, a result of corporate consolidation through mergers and acquisitions over 
a number of years since ownership rules in that sector began to be gradually relaxed towards the end of the Twentieth Century.

Many in the British Community Radio sector (including its representative body, the Community Media Association) recommend a similar approach to the ownership of the new tier of localised DAB multiplexes. Pointing to the protections offered to Community Radio stations, they argue that the multiplexes themselves should be similarly protected against corporate approaches to avoid the creation of networks potentially devoid of local character (CMA 2014: 2).

Early in 2017 , the UK Government successfully piloted legislation through the British Parliament that set out a broad framework for the future licencing of permanent localised DAB multiplexes (DCMS 2017; House of Lords 2017). Much operational detail is missing from this framework, and, at the time of writing, although a DCMS consultation has been run, its conclusions have yet to be published. Once they are, Ofcom intends to consult, in detail, as to how the legislation should be put into a workable regulatory regime that could both serve the interests of smaller-scale broadcasters and ensure the distinctiveness of a new tier of localised DAB multiplexes.

Concerns around ownership and coverage, not least around ensuring effective equivalence of service availability, are just some of the issues that the forthcoming Ofcom consultation is likely to address. As ever there remains a tension between the interests of the small-scale commercial sector and those of the not-for-profit community sector. However, in this particular instance, the potential benefits to both seem to outweigh long standing ideological rivalries, as both sectors suffer the same lack of digital broadcasting opportunities as each other. Both also share concerns that this situation is becoming an increasing disadvantage when compared to the benefits of digital transmission, which larger PSB and commercial stations have been enjoying for some time.

The trial multiplexes carry a range of commercial, non-commercial and new entrant broadcasters and it would seem likely that this is the model that will form the basis of future permanent operations. However, concerns remain about how carrying capacity on these localised multiplexes will be shared. Might, for example, there be reserved capacity for local community radio services, or, indeed, for local commercial stations that otherwise have no access to digital broadcasting platforms?

\section{CONCLUSIONS}

Over the years, wider technological developments, as well as changes in broadcasting policy and regulatory environments, have not made life easy for the proponents of DAB. The consumption of curated audio content is no longer almost entirely the preserve of radio broadcasting alone. Traditional radio has now 
been joined by various alternative "radio-esque" platforms, including streamed audio and podcasts. It could therefore be argued that such developments negate the need to replace traditional analogue broadcasting platforms with a digital alternative of similar ubiquity. However, when it comes to audiences, the consumption of traditional free-to-air radio continues to dominate in terms of absolute audience numbers and listening hours (Ofcom 2016c: 111).

It is now over thirty years since permanent DAB services were first introduced and it does appear that the technology is gradually moving towards the final stage of the hype cycle, within which a "plateau of productivity" may finally be reached. Linked to the hype cycle, "Amara's Law" states that: "We tend to overestimate the effect of a technology in the short run and underestimate the effect in the long run" (Deighton / Kornfeld 2013). It may not yet be possible to conclude that this law applies to DAB, but the original Eureka 147 technical standard has certainly evolved over the years, not least because of the introduction of the enhanced $\mathrm{DAB}+$ standard, which is now beginning to make its presence felt. Arguably however, it is potential changes to the material implementation of DAB that may have as great, or even greater, potential to enhance the practical beneficial impacts of the standard. If this proves to be the case, then the longer-term impacts of DAB will indeed be greater than might have been expected only a few short years ago.

\section{REFERENCES}

Anderson, John Nathan (2013): Radio's Digital Dilemma: Broadcasting in the Twenty-First Century, New York and London: Routledge.

Bower A.J. (1998): “Digital Radio - The Eureka 147 DAB System.” In: Electronic Engineering Magazine April 1998, pp. 55-56. (http://downloads.bbc.co.uk/rd/ pubs/reports/1998-10.pdf).

Clark, Marguerite (1996): “Eureka 147 Continues to Spread.” In: Radio World Magazine 10th July 1996, p. 1.

Coinchon (2010): "Open Software Defined Radio" (Technical Presentation). (https://tech.ebu.ch/events/webinar_opensoftware-definedradio).

Community Media Association (2014): "Response to Ofcom's Small-Scale DAB Trial Consultation (October-December 2014)". (https://www.ofcom.org.uk/_data/assets/pdf_file/0024/74382/community_media_association.pdf).

Crawford, Adam J. (2013): "Profiting from the Hype Cycle." (https://www.caseyresearch.com/articles/profiting-hype-cycle)

Deighton, John/ Kornfeld, Leora (2013): “Amazon, Apple, Facebook, and Google.” Harvard Business School Case 513-060, January 2013. (Revised December 2013.) (http://www.hbs.edu/faculty/ Pages/item.aspx?num=43983). 
DCMS (2010): Digital Radio Action Plan (v1.0). London: Department for Culture Media and Sport.

DCMS (2017): Broadcasting (Radio Multiplex Services) Bill - Explanatory Notes. (https://www.publications.parliament.uk/pa/bills/cbill/2016-2017/0028/ en/17028en.pdf).

EBU (2014): “Digital Radio Toolkit. Key Factors In The Deployment Of Digital Radio.” (https://www.ebu.ch/files/live/sites/ebu/files /Publications/EBU-MIS_ Digital-Radio-Toolkit.pdf)

Eureka (1997): “Eureka-147 - Digital Audio Broadcasting.” (http://www.teilar.gr/ dbData/ProfAnn/profann-1a29fba4.pdf).

Fenn, Jackie/Raskino, Mark (2008): Mastering the Hype Cycle: How to Choose the Right Innovation at the Right Time, Boston: Harvard Business Press.

Gartner Consulting (2017): "Research Methodologies - Gartner Hype Cycle." (http://www.gartner.com/technology/research/ methodologies/hype-cycle.jsp)

Goddard, Grant (2010): DAB Digital Radio Licensed to Fail, London: Radio Books.

Herrmann, Frank/ Erismann Larissa Anna/Prosch, Marcus (2007): “The Evolution of DAB.” In: EBU Technical Review, July 2007 (https://tech.ebu.ch/docs/ techreview/trev_311-dab_dmb.pdf).

HMSO (2004): “Community Radio Order, 2004 (No. 1944) (As Amended).” (http:// www.globalmediapolicy.net/sites/default/files/uksi_20041944_en.pdf).

House of Lords (2017): "Broadcasting (Radio Multiplex Services) Bill (o6th February 2017)". Available on-line at: https://www.publications.parliament.uk /pa/ bills/lbill/2016-2017/0099/17099.pdf).

Kozamernik, Franc (1995): "Digital Audio Broadcasting - Radio Now and for the Future.” EBU Technical Review, Autumn 1995, pp. 2-27. (https://tech.ebu.ch/ docs/techreview/trev_265-kozamernik.pdf).

Lax, Stephen (2003): “The Prospects for Digital Radio Policy and Technology for a New Broadcasting System.” In: Information, Communication \& Society, 6/3, pp. 326-349.

Mustapha, Rashid (2013): "Small Scale DAB. The Potential for Lower-Cost Transmitting Stations in Support of DAB Rollout," (Ofcom Research Document). (https://www.ofcom.org.uk/__data/assets/pdf_file/oo24/ 44808/softwaredab-research.pdf).

NRK (2017): “Radioen Det Første Massemedium [Radio - The First Mass Medium]”. (https://www.nrk.no/organisasjon/radioen---det-forste-massemedium$1.6512029)$.

Ofcom (2010): “The Communications Market: Digital Radio Report (Ofcom's First Annual Digital Progress Report).” (https:// www.ofcom.org.uk/__data/assets/ pdf_file/0020/63191/report210710.pdf).

Ofcom (2012): “DAB Coverage Planning: Report To Government.” (https://www. ofcom.org.uk/__data/assets/pdf_file/0020/3719o/dab_statement.pdf). 
Ofcom (2016a): The Communications Market: Digital Radio Report (Ofcom's Seventh Annual Digital Progress Report). ( https:// www.ofcom.org.uk/__data/ assets/pdf_file/o032/94838/The-Communications-Market-Digital-Radio-Report-2016.pdf).

Ofcom (2016b): “Small Scale DAB Trials - Final Report (26th September 2016)". (https://www.ofcom.org.uk/__data/assets/pdf_file/o021/91371/SSDAB-Finalreport-26-Sep.pdf).

Ofcom (2016c): “The Communications Market 2016: Radio \& Audio." (https:// www.ofcom.org.uk/__data/assets/pdf_file/ooo9/21411/uk_radio.pdf).

OLON (2002): Eureka! Een Oplossing Voor Digitale Kleinschalige Radio, Nijmegen: OLON.

O’Neill, Brian (2009): “DAB Eureka-147: A European Vision for Digital Radio.” In: New Media \& Society 11/1-2, pp. 261-278.

O’Neill, Brian (Editor) (2010): Digital Radio in Europe - Technologies, Industries and Cultures, Bristol: Intellect.

Ray, Bill (2013): “Bloke In Shed Starts Own DAB Radio Station - With Ofcom's Blessing.” (https://www.theregister.co.uk/2013/o8/06/open_source_hacks_ dab_to_the_masses/).

Robjohns, Hugh (2009): “Sounding Off: Is DAB A Disaster?” (http://www.soundonsound.com/people/sounding-dab-disaster)

Sabel, Les (2013): "Overview of the DAB+ System." (https:// www.worlddab.org/ public_document/file/442/DAB__Overview_2013.pdf?1394188480).

Schofield, Jack (2006): “DAB Should Be The New Wave, Not Medium Wave." In: The Guardian 26 October 2006 (https://www.theguardian.com/technology/2006/oct/26/comment.comment).

Spikofski, Gerhard/Klar, Siegfried (2003): “DAB and CD Quality - Reality or Illusion?"In: EBU Technical Review, October 2003. (https://tech.ebu.ch/docs/ techreview/trev_296-dab.pdf).

Sveriges Radio (2008): “Om Sveriges Radio [About Swedish Radio]”. (http:// sverigesradio.se/sida/artikel.aspx?programid=3113\&artikel=1971599)..

Withero, David M. L. / Lavern, Philip A. (1995): “Digital Audio Broadcasting - The Future of Radio." Paper presented at the IBC International Broadcasting Convention, 1995 (http://ieeexplore.ieee.org/document/ 475385/). 